\title{
Tunable chirality of noncentrosymmetric magnetic Weyl semimetals in rare-earth carbides
}

\author{
Rajyavardhan Ray $\mathbb{D}^{1,2 凶}$, Banasree Sadhukhan $\mathbb{D}^{1}$, Manuel Richter $\mathbb{D}^{1,2}$, Jorge I. Facio $\mathbb{D}^{1}$ and Jeroen van den Brink $\mathbb{D}^{1,2,3 凶}$
}

Even if Weyl semimetals are characterized by quasiparticles with well-defined chirality, exploiting this experimentally is severely hampered by Weyl lattice fermions coming in pairs with opposite chirality, typically causing the net chirality picked up by experimental probes to vanish. Here, we show this issue can be circumvented in a controlled manner when both time-reversal- and inversion symmetry are broken. To this end, we investigate chirality disbalance in the carbide family $\mathrm{RMC}_{2}(\mathrm{R}$ a rare-earth and $\mathrm{M}$ a transition metal), showing several members to be Weyl semimetals. Using the noncentrosymmetric ferromagnet $\mathrm{NdRhC}_{2}$ as an illustrating example, we show that an odd number of Weyl nodes can be stabilized at its Fermi surface by properly tilting its magnetization. The chiral configuration endows a topological phase transition as the Weyl node transitions across the Fermi sheets, which triggers interesting chiral electromagnetic responses. Further, the tilt direction determines the sign of the resulting net chirality, opening up a simple route to control its sign and strength.

npj Quantum Materials (2022)7:19; https://doi.org/10.1038/s41535-022-00423-z

\section{INTRODUCTION}

Since their experimental discovery in the TaAs family, Weyl semimetals continue to gain interest. The non-trivial topology of this electronic phase follows from the geometrical properties ${ }^{1}$ associated with electronic bands. Specifically, for band crossing points, a topological invariant can be defined as the flux of Berry curvature through a surface enclosing the point. The low-energy effective theory around such a point corresponds to the Weyl equation ${ }^{2}$, identifying the topological invariant with the corresponding Weyl fermion chirality. In lattice systems, however, Weyl nodes must come in pairs of opposite chirality ${ }^{3}$. Each pair having zero net chirality severely hampers experimental probes sensitive to Brillouin zone integrated quantities from picking up the Weyl node chirality: for this to work, one should create an overall chirality imbalance.

The essential condition to enable the existence of Weyl nodes in the first place is broken spin degeneracy of Bloch states at a generic crystal momentum. This requires time-reversal symmetry $(\Theta)$ or inversion symmetry $(\mathcal{I})$ to be broken. While the first experimental confirmations of topological semimetals were achieved in $\Theta$-symmetric compounds ${ }^{4-11}$, magnetic compounds are naturally appealing due to the broad prospects that the interplay between the electronic structure and external magnetic fields can offer ${ }^{12-22}$. Still, experimentally confirmed magnetic topological semimetals are rather scarce. Two recently investigated cases are the nodal-line semimetal $\mathrm{Co}_{2} \mathrm{MnGa}^{23,24}$ and the Weyl semimetal $\mathrm{Co}_{3} \mathrm{Sn}_{2} \mathrm{~S}_{2}{ }^{25-28}$, both centrosymmetric ferromagnets with non-trivial topology. In $\mathrm{EuCd}_{2} \mathrm{As}_{2}{ }^{29}$ and $\mathrm{EuCd}_{2} \mathrm{Sb}_{2}{ }^{30}$, on the other hand, a Weyl semi-metallic phase is realized for the fully spin-polarized state induced by an external magnetic field.

Here we focus on the rare-earth carbides $R M C_{2}$, with $R$ a rareearth metal and $M$ a transition metal. This broad family of compounds exhibits a diversity of $\Theta$-breaking or $\mathcal{I}$-breaking phenomena ${ }^{31-45}$ both in long-range ordered magnetic compounds $^{31-37}$ as well as in superconducting phases ${ }^{38-41}$ and a complex interplay between these phases and charge density waves $(C D W)^{42-51}$. We show by consideration of available experimental information and own density-functional calculations (DFT) that $\mathrm{RMC}_{2}$ compounds can be categorized in four classes: (I) $\Theta$-symmetric and $\mathcal{I}$-broken semimetals $\left(\mathrm{YCoC}_{2}\right.$ and $\left.\mathrm{LuCoC}_{2}\right)$; (II) $\Theta$ broken and $\mathcal{I}$-symmetric metals $\left(\mathrm{GdRuC}_{2}\right)$; (III) both $\Theta$ - and $\mathcal{I}$-broken semimetals $\left(\mathrm{PrRhC}_{2}, \mathrm{NdRhC}_{2}, \mathrm{GdCoC}_{2}\right.$ and $\left.\mathrm{GdNiC}_{2}\right)$; and (IV) insulators $\left(\mathrm{LaRhC}_{2}\right)$, the latter being of secondary interest for this work. We find that all the mentioned semimetals possess Weyl nodes close to the Fermi energy, as summarized in Table 1, where they also tend to have relatively simple and uncluttered band structures. Figure 1 illustrates the two observed structure types of the (semi-)metallic compounds.

Systems belonging to class III are of particular interest as they allow for specific demonstrations of the unique interplay between topology and magnetism offered by $\mathcal{I}$-broken symmetry. As a proof of principle, we show for $\mathrm{NdRhC}_{2}$ and $\mathrm{GdCoC}_{2}$ how in ferromagnetic (FM) noncentrosymmetric phases tilting of the magnetization $(\mathbf{m})$ along a low-symmetry direction produces a disbalance in the number of opposite chirality Weyl fermions near the Fermi surface: of all Weyl nodes the degeneracy is lifted. Noteworthy, the direction in which the magnetization is canted controls the sign of the chirality disbalance, allowing therefore to switch the dominant low-energy chirality of the electrons. Such chirality imbalanced configuration naturally leads to a variety of physical consequences, some simply related with the chiral symmetry induced on the electronic structure, others with the possibility-unique of chiral systems-of topological phase transitions in which a single Weyl node crosses the Fermi level and moves between regions enclosed by different Fermi surface sheets. We discuss the ramifications of chirality imbalance and the related topological transitions for existence and observation of chiral and gyrotropic magnetic effects (GME) in these compounds.

\footnotetext{
${ }^{1}$ Institute for Theoretical Solid State Physics, Leibniz IFW Dresden, Helmholtzstr. 20, 01069 Dresden, Germany. ${ }^{2}$ Dresden Center for Computational Materials Science (DCMS), TU Dresden, 01062 Dresden, Germany. ${ }^{3}$ Institute of Theoretical Physics, Technische Universität Dresden, 01062 Dresden, Germany. ${ }^{凶}$ email: r.ray@ifw-dresden.de; j.van.den.brink@ifw-dresden.de
} 
R. Ray et al.

Table 1. Symmetry, magnetic ground state and, for the Weyl node closest to the Fermi energy, position, energy ( $(\varepsilon)$, degeneracy (Deg) and band index of the lower branch in the band crossing ( $N$ is the number of valence electrons).

\begin{tabular}{|c|c|c|c|c|c|c|c|c|c|c|c|}
\hline \multirow[t]{2}{*}{ Compound } & \multicolumn{3}{|c|}{ Symmetry } & \multicolumn{3}{|c|}{ Magnetic ground state } & \multicolumn{4}{|l|}{ Weyl nodes } & \multirow{2}{*}{$\begin{array}{l}\mathrm{AHC} \\
\left(\Omega^{-1} \mathrm{~cm}^{-1}\right)\end{array}$} \\
\hline & Class & $\mathcal{I}$ & $\Theta$ & Expt. & OC & $+U$ & $\begin{array}{l}\text { Position } \\
(2 \pi / a, 2 \pi / b, 2 \pi / c)\end{array}$ & $\begin{array}{l}\varepsilon \\
(\mathrm{meV})\end{array}$ & Deg & Band & \\
\hline $\mathrm{LuCoC}_{2}$ & I & - & + & $\mathrm{NM}^{43}$ & NM & NM & $(0.362,0.163,0)$ & 90 & 4 & $N-1$ & 0 \\
\hline $\mathrm{YCoC}_{2}$ & I & - & + & $\mathrm{NM}^{57}$ & NM & NM & $(0.366,0.173,0)$ & 61 & 4 & $N-1$ & 0 \\
\hline $\mathrm{GdCoC}_{2}$ & III & - & - & $T_{\mathrm{C}}=15 \mathrm{~K}^{36}$ & FM & FM & $(0.347,0.206,0.171)$ & 34 & 4 & $N-1$ & 15 \\
\hline $\mathrm{NdRhC}_{2}$ & III & - & - & $\theta_{\mathrm{CW}} \sim 0 \mathrm{~K}^{31,32}$ & FM & FM & $(0.393,0.233,-0.199)$ & 43 & 4 & $N$ & 35 \\
\hline $\mathrm{PrRhC}_{2}$ & III & - & - & $\theta_{\mathrm{CW}} \sim-13 \mathrm{~K}^{31,32}$ & AFM & FM & $(0,0.158,-0.221)$ & 9 & 4 & $N-1$ & -13 \\
\hline
\end{tabular}

Weyl nodes and the anomalous Hall conductivity (AHC) correspond to GGA with open-core (OC) approximation for occupied $4 f$ states. For classes II and III, $\mathbf{m} \|[001]$ was applied. From top to bottom the compounds are presented in order of increasing unit cell volume.

\section{RESULTS AND DISCUSSIONS}

\section{From Y to Lu}

For compounds based on different $\mathrm{R}$ elements, we first present the main structural, electronic and magnetic properties of interest for this work. Our DFT results were obtained using the generalized gradient approximation (GGA) ${ }^{52}$, as implemented in FPLO- $18^{53}$ (https://www.fplo.de/) and considering for the treatment of the occupied $4 f$-states both the open-core approximation (OC) with R-specific $4 f$ spin moment but spherical orbital occupation ${ }^{54}$ and the GGA $+U$ method with the full-localized limit for the double counting correction ${ }^{55}$ and parameters $U=7 \mathrm{eV}$ and $J=1 \mathrm{eV}$. We study compounds which have already been synthesized, including $\mathrm{R}=\mathrm{Y}, \mathrm{La}, \mathrm{Nd}, \mathrm{Pr}, \mathrm{Gd}$ or $\mathrm{Lu}$, and $\mathrm{M}=\mathrm{Co}, \mathrm{Rh}, \mathrm{Ni}$ or $\mathrm{Ru}^{31,32,34,43}$, and use the lattice parameters from The Materials Project ${ }^{56}$ (see the Supplementary Note I for further details). Table 1 includes the available experimental data. For most of the compounds, we find that the DFT calculations agree with the reported magnetic properties. In order to analyze the electronic properties of the whole family on the same footing, we will fix in the following the quantization axis along the direction [001].

We find that the compounds involving rare earths with empty or completely filled $f$-shells, $\mathrm{R}=\mathrm{Y}$, La or Lu, result in $\Theta$-symmetric non-magnetic (NM) states, in agreement with experiment ${ }^{32,43,57}$. Among these, $\mathrm{LaRhC}_{2}$ is the only system that crystallizes in the noncentrosymmetric tetragonal space group $P 4{ }_{1}^{32}$ and displays an insulating electronic structure (see Supplementary Note III). Opposed to this, $\mathrm{YCoC}_{2}$ and $\mathrm{LuCoC}_{2}$ grow in the noncentrosymmetric orthorhombic space group $A m m 2^{43,57}$ and exhibit a semimetallic band structure (see Fig. 2a and Supplementary Note III). Four bands dominate the energy spectrum of $\mathrm{LuCoC}_{2}$ near the Fermi energy. These arise from hybridized Co-3d, C-2p and R-5d states (referred to as $p d$ states from now on). The same characteristic four bands are present at low energy for other compounds with $\mathrm{M}=\mathrm{Co}$ or Rh (Fig. $2 \mathrm{~b}, \mathrm{c}$, e). On the other hand, compounds based on transition metals not isoelectronic to Co, namely, $\mathrm{Ru}$ or $\mathrm{Ni}$, exhibit a shift of the Fermi energy of roughly $\pm 1 \mathrm{eV}$ (Fig. 2d). This shift naturally changes the nature of the bands around the Fermi energy and, as we will see, their electronic properties.

Members of the family based on magnetic $\mathrm{R}$ ions are characterized as a lattice of localized moments on the R- $4 f$ states coupled with the pd states via an onsite (Kondo) exchange. Ruderman-Kittel-Kasuya-Yosida exchange interactions between the $4 f$ moments can lead to long-range magnetic order including the induced moments on the $p d$ states. Consequently, these compounds are an interesting platform to explore how external magnetic fields, which couple primarily with the R- $4 f$ states, can tune Weyl node properties of the low-energy electronic structure associated with the $p d$ states.

Among the magnetic compounds, those obtained by replacing Lu by magnetic rare earths while retaining the space group Amm2 (Fig. 1a, c) belong to Class III. The crystal structure in this case can be regarded as a stack of rare-earth planes, responsible for the breaking of $\Theta$ at low temperatures, and planes of $\mathrm{MC}_{2}$, responsible for the breaking of $\mathcal{I}$.

For these compounds, different magnetic states have been reported. $\mathrm{GdCoC}_{2}$ was first described as antiferromagnetic (AFM) with in-plane moments canted at $48^{\circ}$ from the $a$-axis and $T_{\mathrm{N}}=$ $15.6 \mathrm{~K}^{34}$. However, a more recent study by Meng et al. finds a FM state below $\sim 15 \mathrm{~K}^{36}$. $\mathrm{GdNiC}_{2}$, on the other hand, as most of the $\mathrm{Ni}$ based members in the family ${ }^{35}$, presents an AFM ground state ${ }^{34}$. For $\mathrm{PrRhC}_{2}$ and $\mathrm{NdRhC}_{2}$, experimental data is limited to hightemperature susceptibility measurements ${ }^{32}$. These indicate small Curie-Weiss temperatures $\left(\theta_{\mathrm{CW}} \sim-13 \mathrm{~K}\right.$ and $\sim 0 \mathrm{~K}$, respectively) and magnetic moments in good agreement with the values expected for the trivalent $\mathrm{R}$ ions. $\mathrm{GdRuC}_{2}$ exhibits an interesting contrast to the above, since it presents a transition to a FM phase but the $\mathcal{I}$-breaking distortion in the $\mathrm{MC}_{2}$ layers is absent and the space group is $\mathrm{Cmcm}$ (Fig. 1b), thus belonging to Class $\mathrm{II}^{33}$.

Our DFT results correctly capture the NM states in $\mathrm{YCoC}_{2}$ and $\mathrm{LuCoC}_{2}$ and the FM states in $\mathrm{GdCoC}_{2}$ and $\mathrm{GdRuC}_{2}$. For $\mathrm{NdRhC}_{2}$, we find that both $\mathrm{GGA}+\mathrm{OC}$ and $\mathrm{GGA}+U$ predict an FM ground state. Results for $\mathrm{PrRhC}_{2}$ are somewhat more complex, as GGA+OC and $\mathrm{GGA}+U$ predict as ground state AFM and FM order, respectively. The only clear deviation from experiment is found in $\mathrm{GdNiC}_{2}$, where our calculations predict the ground state to be FM. One should, however, keep in mind that in the Ni-based carbides, a strong interplay between the magnetic state and a CDW (not explored in our calculations) has been established. In fact, there are indications that the CDW tends to compete against the FM phase ${ }^{42,46,48,50}$. Specifically in $\mathrm{GdNiC}_{2}$, different metamagnetic transitions have been observed under moderate external magnetic fields, yielding an interesting and complex phase diagram $^{42,45,51}$.

\section{Weyl nodes}

We now turn our attention to the topological properties of the electronic structure. For this, we will focus on the GGA + OC calculations. As a common reference for the following, we define for each compound the number of valence electrons as $N$ and search for Weyl points in a relevant low-energy window [-120, $120] \mathrm{meV}$, using the PYFPLO module of the FPLO package (). 

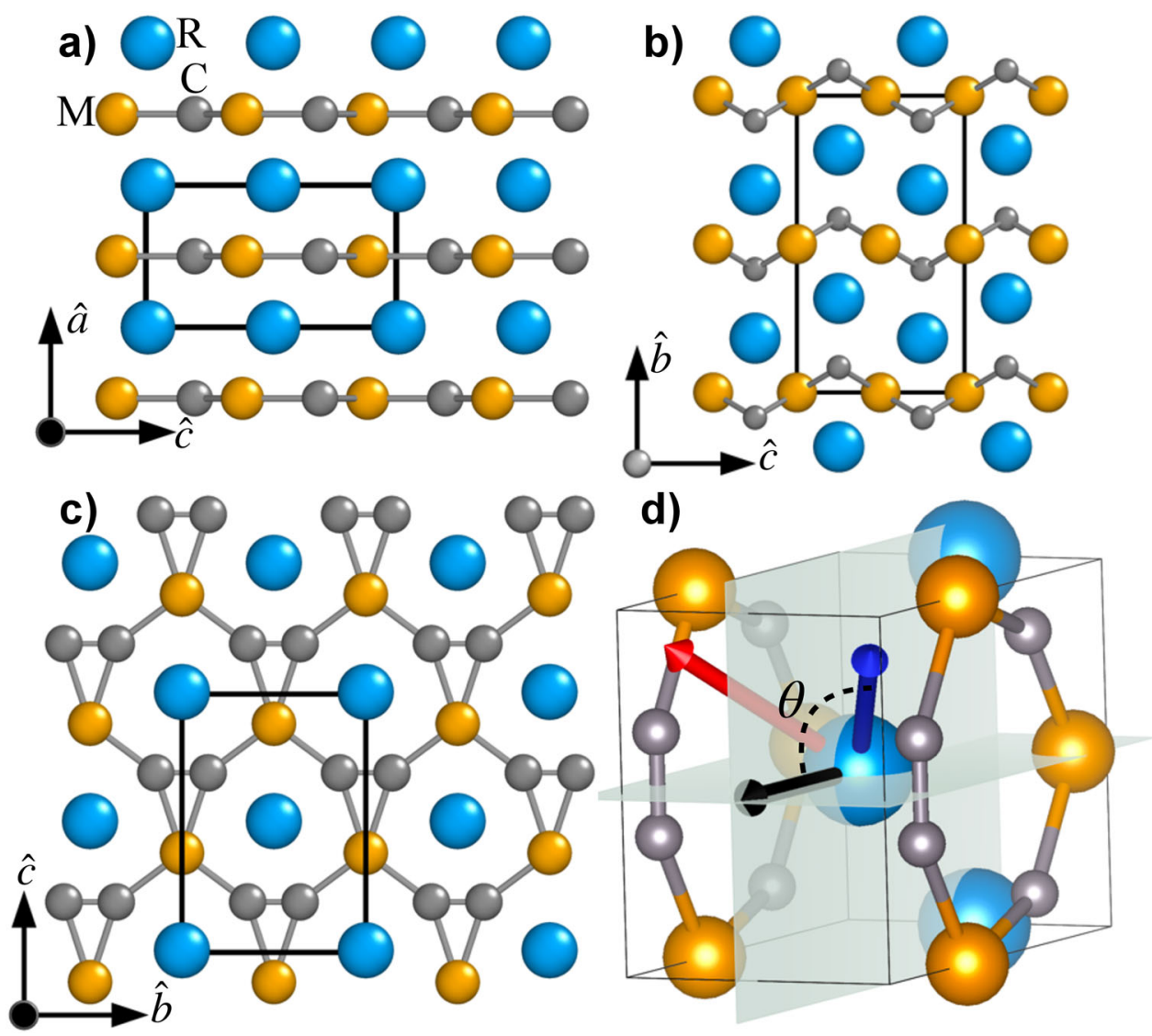

Fig. 1 Crystal structures of $\mathbf{R M C}_{\mathbf{2}}$. $\mathbf{a}, \mathbf{c}, \mathbf{d}$ Compounds with noncentrosymmetric Amm2 space group, and $\mathbf{b} \mathrm{Cmcm}$ space group. $\mathbf{a}$, $\mathbf{b}$ illustrate the layered structure, formed by a quasi-planar network spanned by the transition metal carbide complex, $\mathrm{MC}_{2}$, while the rare-earth ions, $\mathrm{R}$, occupy the interstitial space between the layers. In d, reflection symmetry planes are depicted in green while the arrows indicate directions [001] (black), [111] (red) and [-111] (blue). The conventional unit cells are indicated by black solid lines.

Figure $2 \mathrm{~g}$ shows the energy of the identified Weyl nodes for all the compounds considered in this work, ordered as a function of their unit cell volume $(V)$. Table 1 includes the position and energy of the respective lowest energy Weyl nodes (for a complete list, see Supplementary Note III). Weyl nodes at low energy are found both for $\Theta$-symmetric and $\Theta$-breaking cases and, among the latter, both for compounds with FM or AFM orderings. $\mathrm{YCoC}_{2}$ has been previously studied in ref. ${ }^{57}$ and our results agree with the addition that the search for crossings between bands $N-1$ and $N$ discloses Weyl nodes at even lower energy, indicated in Fig. $2 \mathrm{~g}$.

Relations between the Weyl node structure of different compounds can also be established. For instance, an inspection of the node coordinates reveals that the fourfold degenerate Weyl nodes of lowest energy in $\mathrm{YCoC}_{2}$, which lie in the $k_{z}=0$ plane, are also present in $\mathrm{LuCoC}_{2}$ but are higher in energy. It further suggests that on replacing $\mathrm{Y}$ by $\mathrm{Gd}$, the large exchange field induced by the Gd-4f spins moves these Weyl nodes away from the $k_{z}=0$ plane and to a lower energy. To confirm this, we carried out explicit computation of Weyl points in $\mathrm{YCoC}_{2}$ under external magnetic field acting on the spin degrees of freedom. Indeed, we find that the field endows the nodes a finite $k_{z}$ component.

Regarding $\mathrm{GdNiC}_{2}$ and $\mathrm{GdRuC}_{2}$, the Fermi energy shift associated with their different number of valence electrons with respect to $\mathrm{GdCoC}_{2}$ is naturally in opposite directions and therefore has different consequences. On $\mathrm{GdRuC}_{2}$, it increases the density of states and the complexity of the low-energy band structure. While it is interesting that it presents Weyl nodes close to the Fermi surface, it should not be considered as a semimetal. On the other hand, the upward shift of Fermi energy makes the Ni-based compound of strong interest. Indeed, upon this shift, a single pair of Weyl nodes - the minimum possible in a periodic system-lies $\sim 100$ meV above the Fermi energy. Our calculations performed on different compounds neatly explain the origin of these Weyl nodes: the isostructural but $\mathrm{NM} \mathrm{LuCoC}_{2}$ features twofold degenerate bands along the line $X-T$ forming a massive Dirac cone $\sim 1 \mathrm{eV}$ above the Fermi level (Fig. 2a). The band degeneracy is protected by a reflection symmetry and is lifted in the isolectronic FM compounds (Fig. 2c, e), where the Dirac cone is split into Weyl cones. Substituting $\mathrm{Co}$ by $\mathrm{Ni}$ shifts these Weyl nodes closer to the Fermi level, while keeping them along $X-T$ (Fig. 2d). While the FM phase in $\mathrm{GdNiC}_{2}$ has only been stabilized with an external magnetic field ${ }^{42,45}$, it could be interesting to study this phenomenology in $\mathrm{SmNiC}_{2}$, where the competing $\mathrm{CDW}$ is suppressed $^{40}$ leading to a FM ground state ${ }^{35}$.

The rather large volume change caused by the substitutions $\mathrm{Gd} \rightarrow \mathrm{Nd}$ or $\mathrm{Pr}$ and $\mathrm{Co} \rightarrow \mathrm{Rh}$ naturally induces sizable changes in the electronic structure which remains, however, semi-metallic in $\mathrm{NdRhC}_{2}$ and $\mathrm{PrRhC}_{2}$. Also, low-energy Weyl nodes are present in these compounds (Fig. 2g). While there are already examples of noncollinear AFM Weyl semimetals ${ }^{58}$, should the predicted magnetic structure of $\mathrm{PrRhC}_{2}$ be confirmed, this compound would be (to the best of our knowledge) the first realization of a collinear AFM Weyl semimetal. In addition, it would allow for the presence of the recently uncovered crystal Hall effect ${ }^{59}$. This effect is characterized by a finite anomalous Hall conductivity (AHC) in a collinear antiferromagnet due to the asymmetric local environment of the magnetic sublattices. Our AHC calculations (see Table 1) show that this effect is indeed present in $\mathrm{PrRhC}_{2}$. 

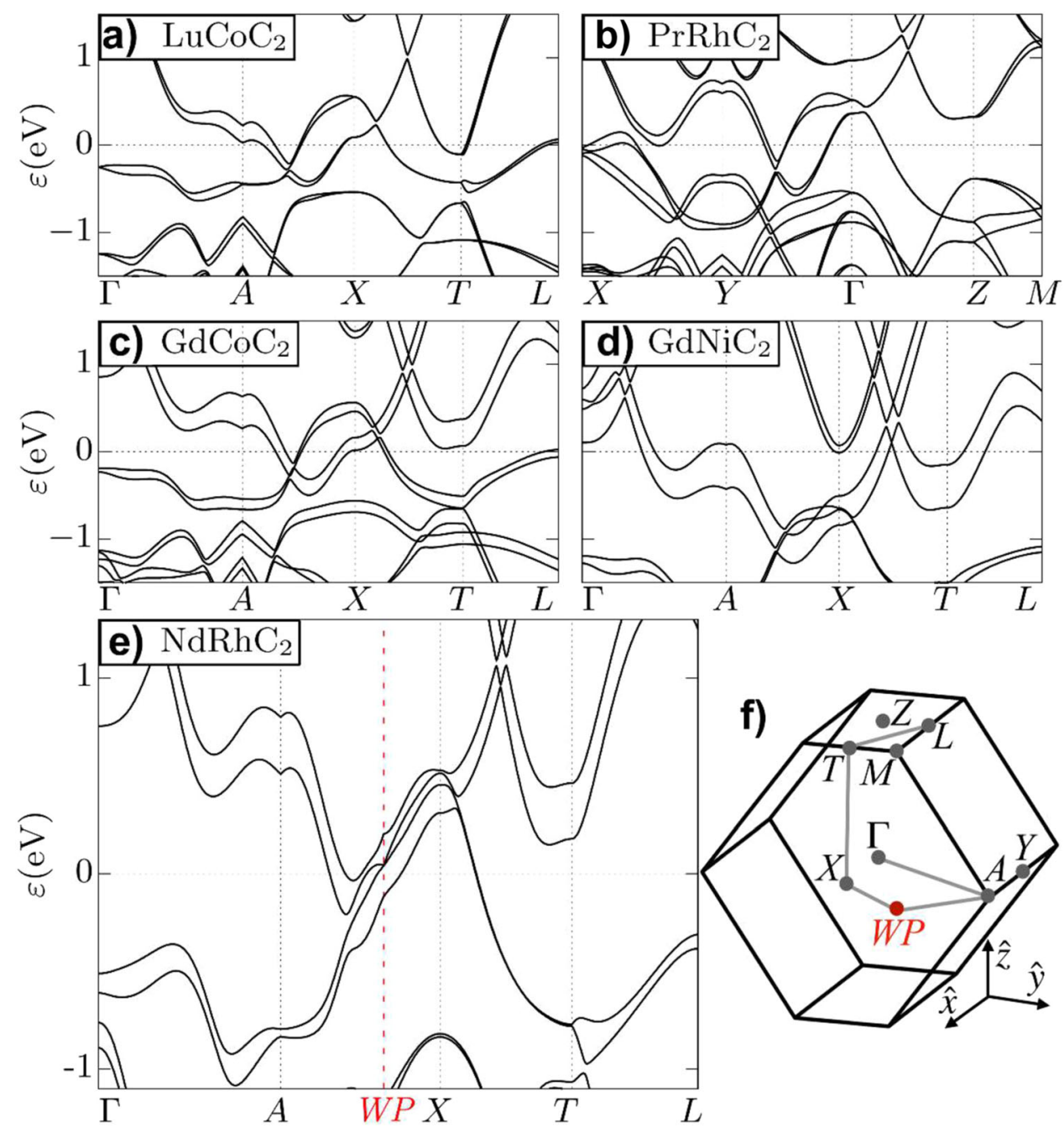

g)

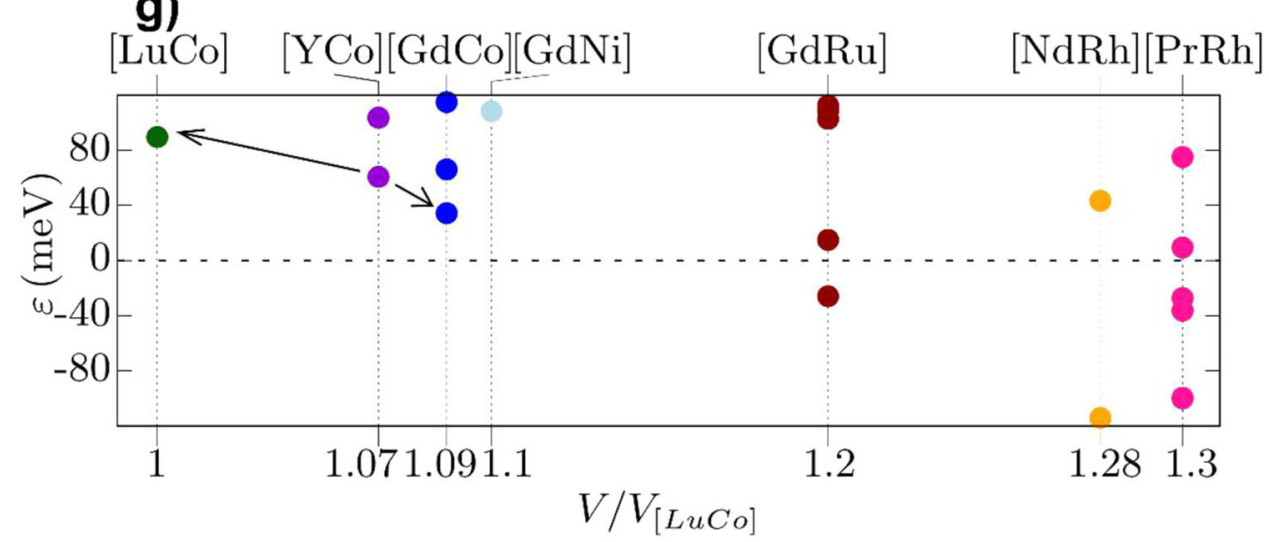

Fig. 2 Electronic properties. a-e Band structures of representative rare-earth dicarbide compounds. $\mathbf{f}$ Brillouin zone. The red point corresponds to the Weyl node closest to the Fermi energy for $\mathrm{NdRhC}_{2}$ and has coordinates $(0.665,0.309,-0.189) \AA^{-1}$. For $\mathbf{b}$, the chosen highsymmetry points differ due to the AFM doubling of the unit cell. $\mathbf{g}$ Energy of the Weyl nodes vs. unit cell volume for all compounds considered in this work.

\section{Pumping chirality to and through the Fermi surface}

Due to the Nielsen-Ninomiya "no-go" theorem for chiral lattice fermions ${ }^{3,60}$, Weyl nodes come in pairs of opposite chirality ${ }^{61}$ (which can be broken up by very strong electron-electron interactions ${ }^{62,63}$ ). In a crystal, Weyl nodes occur in multiplets, their degeneracy being dictated by the Shubnikov group of the material which relates Weyl nodes of same or different chirality.
Depending on the specific Shubnikov group, the Weyl node degeneracy need not be even. If the degeneracy is odd, pairs of opposite chirality are necessarily energy-split. The energy splitting between nodes of opposite chirality, a key quantity for the magnitude of different electromagnetic responses sensitive to the Weyl node chirality, actually depends on the material and external conditions. Low symmetry, in particular the absence of inversion 
a)

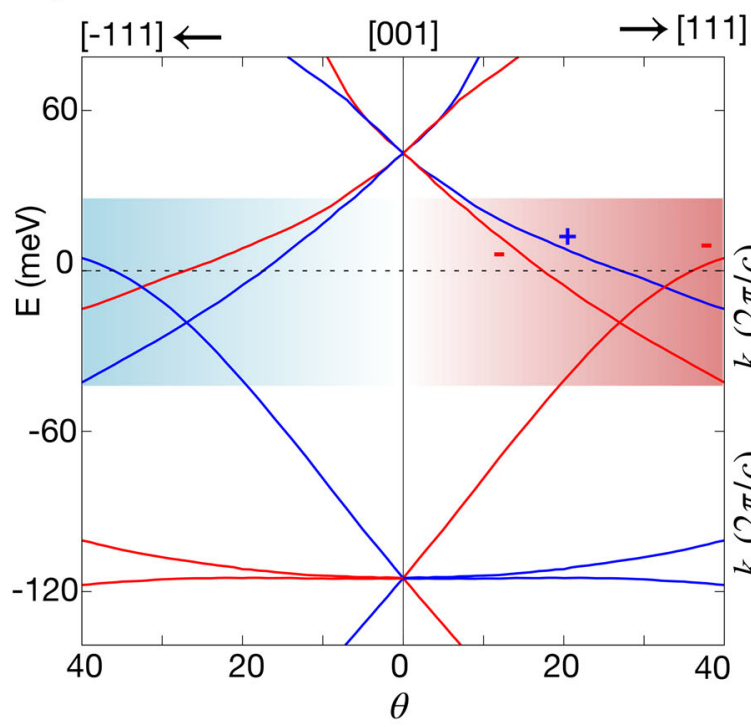

b)

b)

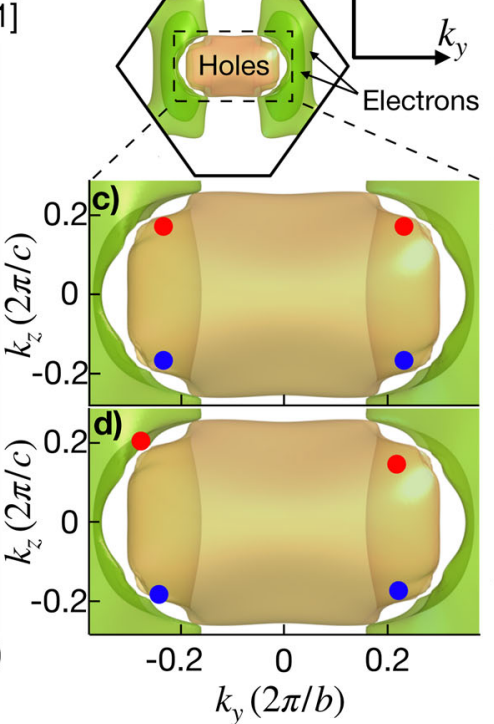

e)

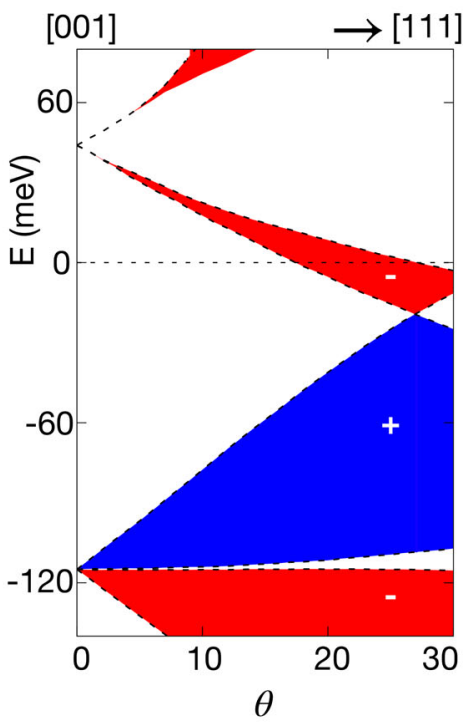

Fig. 3 Pumping chirality to and through the Fermi surface. a Energy of Weyl nodes in $\mathrm{NdRhC}_{2}$ as a function of the canting angle $\theta$. Starting from the magnetization along the direction [001], on the left the canting is toward [-111], while on the right is toward [111]. Blue and red correspond to Weyl nodes of positive and negative chirality, respectively. The colored region highlights a wide angle range in which near the Fermi surface Weyl fermions of one chirality are majority. b Bulk Fermi surface for $\theta=0$. c Zoom into the Fermi surface showing the Weyl nodes closest to it for $\theta=0$. A Weyl node quartet places inside a hole pocket. $\mathbf{d}$ Same for $\theta \sim 20$, where now a single Weyl node has moved into an electron Fermi surface, leaving a net chirality in the hole pocket. e Phase diagram in the space of energy and canting angle indicating the regions in which the hole pocket carries a net Chern number.

and mirror symmetries is of the essence. Here we build on the idea of using the magnetic degrees of freedom in a $\mathcal{I}$-broken material to reduce the symmetry such that the Shubnikov group contains only the identity.

As a proof of principle, we consider $\mathrm{NdRhC}_{2}$, although the physics we discuss can be readily extended to other noncentrosymmetric magnetic compounds. In the FM ground state, $\mathbf{m}$ points along the [001] direction and the Shubnikov group contains $\left\{E, m(x) \Theta, m(y) \Theta, C_{2}(z)\right\}$, where $m$ and $C_{2}$ represent the mirror symmetries and the twofold rotation, respectively. Therefore, for each Weyl node away from high-symmetry lines, there are three degenerate symmetry-related partners. Any component of $\mathbf{m}$ along a low-symmetry direction does not only break the rotation $C_{2}(z)$ but also the symmetries involving mirrors. Thus, starting from $\mathbf{m}$ along [001], a perturbation that cants $\mathbf{m}$ toward, e.g., [111] leaves $\{E\}$ as the only symmetry element, removing all degeneracies among the Weyl nodes. The natural question is how large this effect is.

Figure 3a shows the energy of Weyl nodes in $\mathrm{NdRhC}_{2}$ as $\mathbf{m}$ is canted at an angle $\theta$ toward [-111] (on the left) or [111] (right). At $\theta=0$, there are two sets of fourfold degenerate Weyl nodes in the energy range $[-120,80] \mathrm{meV}$. Remarkably, even a moderate canting of $\mathbf{m}$ is enough to produce experimentally meaningful energy splittings between Weyl nodes of opposite chirality, of the order of tens of meV. We have verified that similar results are obtained using either the experimentally reported crystal structures $^{32}$ or crystal structures relaxed keeping the ratio of the lattice constants as in the experimental structure (see Supplementary Note II).

The tuning of Weyl nodes to the Fermi surface at specific angles resembles the prediction for $\mathrm{CO}_{3} \mathrm{Sn}_{2} \mathrm{~S}_{2}{ }^{17}$, with the difference that in the latter case the crystal structure is centrosymmetric. This difference is crucial as $\mathcal{I}$ always connects Weyl nodes of opposite chirality and, therefore, enforces a vanishing total chirality of the Weyl nodes at the Fermi surface. While mirror symmetries can be reversibly broken by external magnetic fields, breaking $\mathcal{I}$ is the structural prerequisite for tuning an odd number of Weyl nodes to the Fermi surface.

The two chosen canting directions are related by a crystal mirror symmetry (Fig. 1d), and therefore, the chirality of the Weyl nodes reaching the Fermi surface at a certain $\theta$ is opposite for [-111] and [111]. Thus, the canting direction provides an experimentally viable way of discarding effects not originated in the chirality disbalance. It is worth noting that this chirality valve is effective not only at the specific angle at which a Weyl node crosses the Fermi surface. Indeed, due to the particularly large energy trajectory of one of the nodes below the Fermi energy, in a wide range of angles for $\theta \gtrsim 20^{\circ}, \mathrm{NdRhC}_{2}$ exhibits two Weyl fermions of negative (positive) and only one of positive (negative) chirality in a transport-relevant energy window of $\pm 40 \mathrm{meV}$ around the Fermi energy for $\theta$ toward [111] ([-111]).

\section{Magnetism-induced bulk topological phase transitions}

The dynamics of a single Weyl node going from above to below the Fermi level is a topological phase transition which can be described by the flux of the Berry curvature through the different Fermi surface sheets:

$$
C_{n a}=\frac{1}{2 \pi} \int_{a} d S \cdot \Omega_{n k}
$$

where $a$ denotes different sheets of the Fermi surface associated with the band $n . C_{n a}$ can be computed based on the momentum position of the Weyl nodes connecting the bands $n$ and $n+1$ and the bands $n$ and $n-1^{64}$. The latter are found to play no role in the energy and angle range analyzed here. In the following we will focus on only one band so we drop the $n$ index. In $\mathrm{NdRhC}_{2}$, two electron-like and two hole-like bands conform the Fermi surface, shown in Fig. 3b. For $\theta<\theta_{c 1} \sim 20^{\circ}$, the four Weyl nodes above the Fermi energy at $\theta=0$ lie inside the outer hole pocket which has, therefore, $C_{\text {hole }}=0$ (Fig. $3 c$ ). At $\theta=\theta_{c 1}$, one Weyl node sits exactly on the Fermi surface. This is a type-II node connecting the outer hole pocket with an electron Fermi surface (Fig. 3d). Further increasing $\theta$, this Weyl node goes below the Fermi level and leaves 
the hole pocket. In this situation, $\left|C_{\text {hole }}\right|=1$. This holds for an angle range between $\theta_{c 1}$ and $\theta_{c 2} \sim 27^{\circ}$, since at $\theta_{c 2}$ another Weyl node of opposite chirality to the previous one transitions out of the hole pocket. As a result, in the parameter space of canting angle $\theta$ and chemical potential, the system presents a series of topological phase transitions in which the topological invariant $C_{\text {hole }}$ changes (Fig. 3e).

\section{Magnetic anisotropy and Weyl node dynamics}

A relevant question is how large is the magnetic field required to cant the magnetization in order to enable the Weyl node dynamics described above. Within the OC approximation employed here, the contribution of the $4 f$ states to the magnetic anisotropy energy (MAE) is not considered. Our estimates for the MAE arising from the remaining valence electrons suggest that the first topological phase transition is accessible at $\sim 5 \mathrm{~T}$. An order of magnitude estimate for the $4 f$ contributions can be obtained from the experimental anisotropy field of the well-known permanent magnet compound $\mathrm{Nd}_{2} \mathrm{Fe}_{14} \mathrm{~B}$ in comparison with the anisotropy field of $\mathrm{Y}_{2} \mathrm{Fe}_{14} \mathrm{~B}$. The former has an anisotropy field of about $\sim 7 \mathrm{~T}$ at room temperature ${ }^{65}$, the latter somewhat $<3 \mathrm{~T}^{66}$, implying similar magnitude of the $\mathrm{Nd}-4 f$ and the non- $4 f$ contributions.

Figure 4 shows the energy of the Weyl nodes closest to the Fermi energy in $\mathrm{GdCoC}_{2}$ as the magnetization is canted. As in the $\mathrm{Nd}$-based compound, the induced energy splitting between Weyl nodes can reach tens of meV. This is interesting since in this compound the $4 f$-contribution to the MAE vanishes to first-order in the spin-orbit coupling. These results are in line with previous work where it has been shown that the energy scale associated with the change in energy of the Weyl nodes due to canting of the magnetization in general does not scale with the $M A E^{17}$. Last, notice that the larger magnetic moment of $\mathrm{Gd}^{3+}$ contributes to lowering the required magnetic field to cant the magnetization.

\section{Discussion}

The predicted Weyl nodes dynamics has consequences on a number of physical observables. Some of them are related to the effects that canting of the magnetization has on the symmetry of the electronic structure, others follow from the topological phase transitions associated with having a single Weyl node going through the Fermi surface. We now briefly outline these open possibilities.

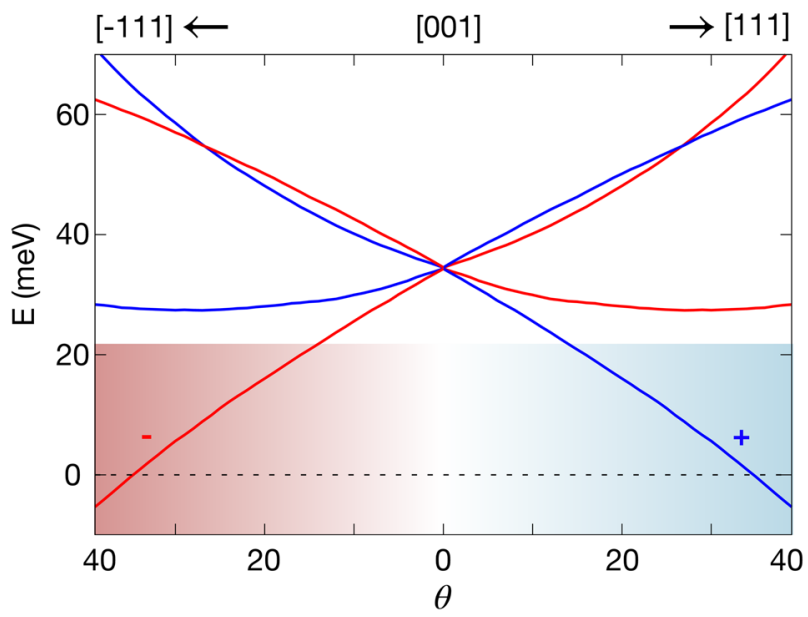

Fig. 4 Chirality imbalance in $\mathrm{GdCoC}_{2}$. Energy of the Weyl nodes as a function of the canting angle $\theta$ for $\mathrm{GdCoC}_{2}$. Blue and red lines, respectively, correspond to Weyl nodes of positive and negative chirality.
The bulk Fermi surface topological phase transitions lead to visible consequences on the surface electronic structure. To illustrate this, we consider the (100)-surface of $\mathrm{NdRhC}_{2}$ terminated on a $\mathrm{RhC}_{2}$ plane. In addition to the corresponding projection of the bulk Fermi surface, the surface Fermi surface exhibits clear Fermi arcs connecting the projection of opposite chirality Weyl nodes, as shown in Fig. 5. For $\theta=0$, Fig. $5 b$ shows the Fermi arcs fixing the Fermi energy at the Weyl node energy $\left(\varepsilon_{\mathrm{F}}=0.043 \mathrm{meV}\right)$, while Fig. $5 c$ corresponds to the charge-neutrality chemical potential $\left(\varepsilon_{\mathrm{F}}=0\right)$. As one of the Weyl nodes moves out from the hole pocket, the upper part of the Fermi arc, which at $\theta=0$ starts in the hole pocket (Fig. $5 \mathrm{~d}$ ), at $\theta=25^{\circ}$, starts in the electron Fermi surface (Fig. $5 d$ ). We notice that similar results are obtained for an Nd-terminated surface.

The GME accounts for the generation of an electrical current by a time-dependent magnetic field and can be regarded as the lowfrequency limit of the natural optical activity in inversion-broken materials ${ }^{67,68}$ :

$j_{i}=a_{i j}^{\mathrm{GME}} B_{j}$.

The tensor $a_{i j}^{\mathrm{GME}}$ is determined by the angular momentum of the Bloch states at the Fermi surface, and the chiral symmetry is a sufficient condition for it to be nonzero ${ }^{67}$. Thus, as explained earlier, canting of the magnetization along a low-symmetry direction, which breaks all the mirror symmetries, together with the inversion-broken lattice structure enable the GME in the carbides family. When evaluated for simplified models for Weyl semimetals, the GME is found to scale linearly with the energy splitting between Weyl nodes ${ }^{67}$. While the question of how such results are modified when having more realistic band structures deserves further investigation, the sizable and controllable energy splitting here predicted both for $\mathrm{NdRhC}_{2}$ and $\mathrm{GdCoC}_{2}$ makes them interesting platforms to explore the GME.

The chiral magnetic effect (CME) also accounts for the generation of an electrical current under applied magnetic field but is fundamentally different to the GME in that it is driven by topological properties of the Fermi surface ${ }^{69}$. Specifically, the relevant topological invariants are the Chern numbers associated with the Fermi surfaces (Eq. (1)). In the presence of electric and magnetic fields having a common projection $\mathbf{E} \cdot \mathbf{B}$, the $C_{a}$ invariants break the conservation of the electron density $n_{i}$ of individual Fermi surface sheets. Specifically, the continuity equation describing the conservation of charge reads:

$\frac{\partial n_{a}}{\partial t}+\nabla \cdot \mathbf{j}_{a}=\frac{C_{a}}{4 \pi^{2}} \mathbf{E} \cdot \mathbf{B}$

As shown in ref. ${ }^{69}$, a natural consequence of nonzero $C_{\alpha}$ invariants is the CME. Specifically, turning on a $\mathbf{E} \cdot \mathbf{B}$ for a finite time causing a difference $\Delta \mu$ between the chemical potential of sheets having opposite Chern number $C$ leads to a finite current $j=C \Delta \mu B / 4 \pi^{2}$. Remarkably, as indicated by Fig. 3e, canting of the magnetization in noncentrosymmetric magnets can provide a way to turn on/off the CME. In particular, when a type-II Weyl node crosses the Fermi energy, as predicted here for $\mathrm{NdRhC}_{2}$ and $\mathrm{GdCoC}_{2}$, the switching on/off of the CME directly exposes the underlying Weyl node dynamics.

Last, we emphasize that although both the GME and the CME rely on the system having a chiral Shubnikov group, the requirements for the $\mathrm{CME}$ are stronger as also a finite Berry curvature flux trough the Fermi surfaces sheets is necessary. This distinction suggests that the dependence of the GME and of the CME on the canting angle may be qualitatively different, the latter including rather abrupt changes according to the phase diagram in Fig. 3e.

To summarize, realization of a chirality imbalance configuration in real materials is of paramount importance for exploiting the most essential property of Weyl fermions, their chirality. Our findings not only show that the family of carbides with broken $\mathcal{I}$ 

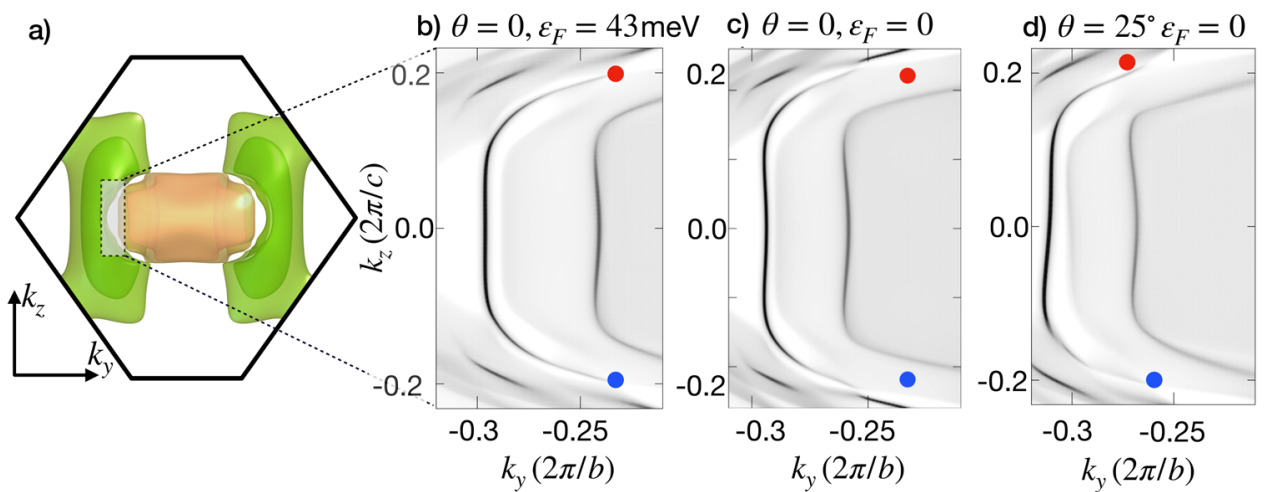

Fig. 5 Fermi arcs. a Bulk Fermi surface of $\mathrm{NdRhC}_{2}$. b Fermi surface of the $\mathrm{RhC}_{2}$-terminated (100)-surface without canting of the magnetization, fixing the Fermi energy at the Weyl node energy $\left(\varepsilon_{F}=43 \mathrm{meV}\right)$. c Same for Fermi energy at the charge-neutrality chemical potential $\left(\varepsilon_{F}=0\right)$. $d$ Same as c for a canting angle $\theta=25^{\circ}$.

a)
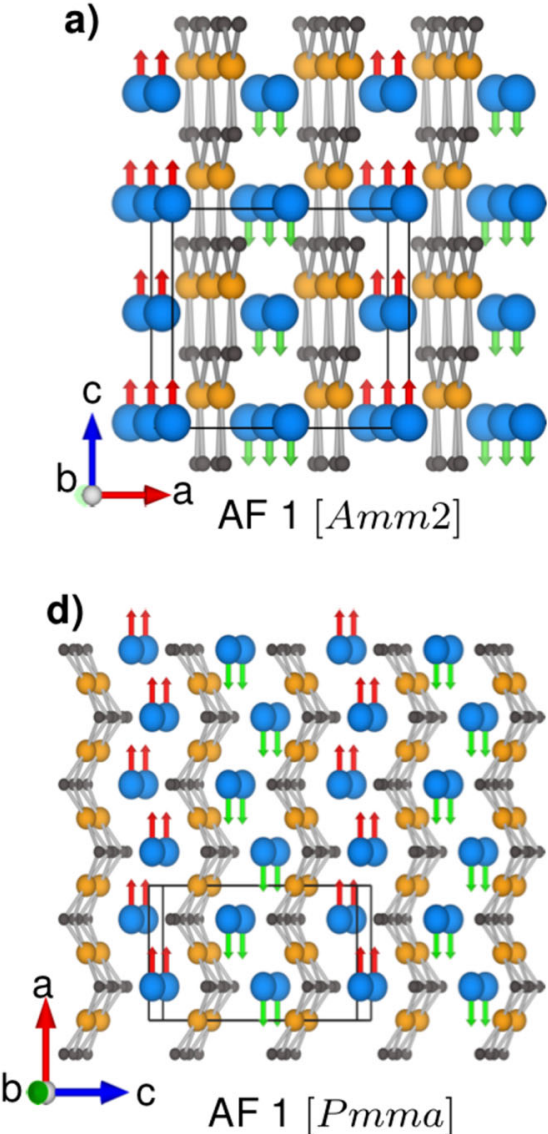

b)

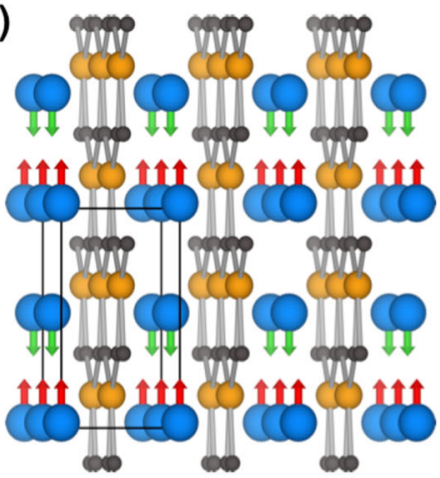

AF $2[P m m 2]$ e)

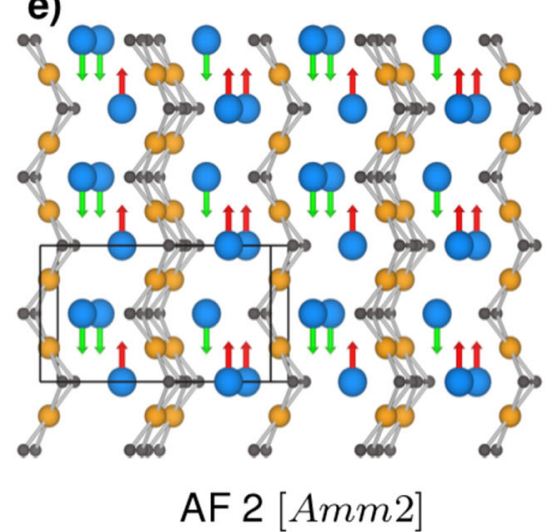

c)

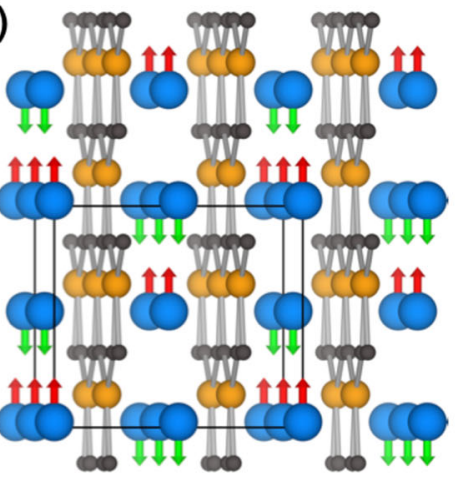

AF $3[\operatorname{Imm} 2]$

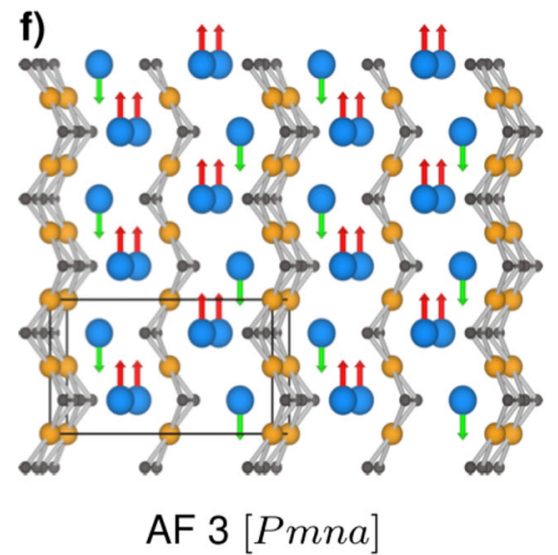

Fig. 6 Magnetic configurations. AFM configurations considered for compounds belonging to a-c the symmetry classes III and $\mathbf{d}-\mathbf{f}$ the symmetry class II. The rare earth atoms, transition metal atoms and carbon atoms are, respectively, shown with blue, yellow and gray spheres. The relative spin directions on the $\mathrm{R}$ atoms are represented by arrows. The choice of quantization axis is only schematic. For each case, the resulting lower-symmetry space groups are also indicated.

and broken $\Theta$ are natural candidates to that end, but also that canting of the magnetization toward a low-symmetry direction in inversion-broken magnets can be generally considered as a knob to measure and control various chiral electromagnetic responses, particularly of gyrotropic and chiral magnetic type.

These effects were recently shown to harbor particular application potential: as our asymmetric Weyl semimetals from the $\mathrm{RMC}_{2}$ carbide family naturally have a chirality dependent
Fermi velocity of Weyl cones, time-dependent pumping of electrons from a non-chiral external source can be used to generate a non-vanishing chiral chemical potential. This again generates, via the chiral anomaly, a current along the direction of an applied magnetic field even in the absence of an external electric field so that the material acts as a rectifying element ${ }^{70}$. Moreover, chirality imbalanced has been predicted to trigger an interesting photoresponse. The simultaneously broken particle- 
hole and spatial inversion symmetry can generate giant photocurrents, suggesting a potential application of these asymmetric Weyl semimetals for creating tuneable $\mathrm{THz}$ photosensors ${ }^{71}$.

\section{METHODS}

\section{Band structure calculations}

DFT calculations were carried out using the Perdew-Burke-Ernzerhof implementation ${ }^{52}$ of the GGA using the full-potential local-orbital (FPLO) code $^{53}$, version 18.00-57 (https://www.fplo.de). A k-mesh with $12 \times 12 \times$ 12 subdivisions was used for numerical integration in the Brillouin zone along with a linear tetrahedron method. Spin-orbit effects were included in the self-consistent calculations via the 4-spinor formalism implemented in the FPLO code.

The description of the $4 f$ elements within the framework of DFT is still a subtle problem ${ }^{54}$. In this work, we considered both of the widely-used approaches to treat the $4 f$-shell: the OC approximation, and the GGA $+U$ scheme. In the first approach, we set the spin for $\mathrm{R}^{3+}$ ions according to the Hund's first rule while maintaining a spherically averaged distribution of electrons in the $4 f$ shell ${ }^{54}$. The trivalent configuration of the rare-earth atoms is found in the related Ni-based carbides $\mathrm{RNiC}_{2}(\mathrm{R}=\mathrm{Pr}, \mathrm{Nd}, \mathrm{Gd})^{34,72}$ and $\mathrm{GdCoC}_{2}{ }^{36}$. In the second approach, to circumvent the usual problem of multiple metastable solutions, we used the occupation matrix control ${ }^{73,74}$, whereby several different initial $4 f$ density matrices, corresponding to +3 valence of $\mathrm{R}$ ions, were considered to explore the energy landscape. We used the full-localized limit of the double counting scheme with $F^{0}=$ $7.0 \mathrm{eV}, F^{2}=11.92 \mathrm{eV}, F^{4}=7.96 \mathrm{eV}$, and $F^{6}=5.89 \mathrm{eV}$, leading to $U=7 \mathrm{eV}$ and $J=1 \mathrm{eV}$. For most of the compounds, both GGA + OC and GGA $+U$ favors the magnetic moments to be in the rare-earth planes. Therefore, we consider $\mathbf{m} \|[001]$ for all the compounds for brevity and comparison. While we can anticipate that the existence of Weyl nodes at low energies is a finding robust to these explored choices, we will discuss to what extent details such as position in energy and momentum can be affected in a forthcoming publication.

\section{Topological electronic properties}

To study the topological properties, we constructed a tight-binding model based on maximally projected Wannier functions. In the basis set, states lying in the energy window $-9.5-10 \mathrm{eV}$ were considered. The Wannier basis set typically included the $5 d$ and $6 s$ states for R (respectively, $4 d$ and $5 s$ for $\mathrm{Y}$ ), valence $d$ and $s$ states for $\mathrm{M}$, and $2 s$ and $2 p$ states for $\mathrm{C}$. The accuracy of the resulting tight-binding models was typically $\lesssim 15 \mathrm{meV}$ compared to the self-consistent band structures.

\section{Magnetic configurations}

To study the relative stability of different collinear long-range ordered states (applicable to materials belonging to classes II and III), different lower-symmetry AFM configurations were generated:

- AF1, with AFM interlayer and ferromagnetic (FM) intralayer couplings.

- AF2, with FM interlayer and AFM intralayer interactions.

- AF3, with AFM interlayer as well as AFM intralayer interactions.

Figure 6 shows these AFM configurations for compounds belonging to classes II and III.

\section{DATA AVAILABILITY}

The data supporting the present work are available from the corresponding author(s) upon request.

\section{CODE AVAILABILITY}

The data presented in the manuscript were obtained using the Full-Potential LocalOrbital (FPLO) code. For details, please see: https://www.fplo.de/. The raw data were subject to basic post-processing (e.g. sorting) using bash/python scripts. All relevant data points were included.

Received: 23 March 2021; Accepted: 7 January 2022; Published online: 09 February 2022

\section{REFERENCES}

1. Berry, M. V. Quantal phase factors accompanying adiabatic changes. Proc. R. Soc. Lond. A Math. Phys. Sci. 392, 45-57 (1984).

2. Weyl, H. Elektron und gravitation. I. Z. Phys. 56, 330-352 (1929).

3. Nielsen, H. B. \& Ninomiya, M. No-go Theorum for Regularizing Chiral Fermions. Tech. Rep. (Science Research Council, United Kingdom, 1981).

4. Xu, S.-Y. et al. Discovery of a Weyl fermion semimetal and topological Fermi arcs. Science 349, 613-617 (2015).

5. Yang, L. et al. Weyl semimetal phase in the non-centrosymmetric compound TaAs. Nat. Phys. 11, 728-732 (2015).

6. Lv, B. Q. et al. Experimental discovery of Weyl semimetal TaAs. Phys. Rev. X 5, 031013 (2015).

7. Di-Fei, X. et al. Observation of Fermi arcs in non-centrosymmetric Weyl semimetal candidate NbP. Chin. Phys. Lett. 32, 107101 (2015).

8. Souma, S. et al. Direct observation of nonequivalent Fermi-arc states of opposite surfaces in the noncentrosymmetric Weyl semimetal NbP. Phys. Rev. B 93, 161112 (2016).

9. Xu, S.-Y. et al. Experimental discovery of a topological Weyl semimetal state in TaP. Sci. Adv. 1, e1501092 (2015).

10. $\mathrm{Xu}, \mathrm{N}$. et al. Observation of Weyl nodes and Fermi arcs in tantalum phosphide. Nat. Commun. 7, 11006 (2016).

11. Haubold, E. et al. Experimental realization of type-ll Weyl state in noncentrosymmetric TalrTe 4 . Phys. Rev. B 95, 241108 (2017).

12. Burkov, A. A., Hook, M. D. \& Balents, L. Topological nodal semimetals. Phys. Rev. $B$ 84, 235126 (2011).

13. Hirschberger, M. et al. The chiral anomaly and thermopower of Weyl fermions in the half-Heusler GdPtBi. Nat. Mater. 15, 1161-1165 (2016).

14. Cano, J. et al. Chiral anomaly factory: creating Weyl fermions with a magnetic field. Phys. Rev. B 95, 161306 (2017).

15. Borisenko, S. et al. Time-reversal symmetry breaking type-Il Weyl state in $\mathrm{YbMnBi}_{2}$. Nat. Commun. 10, 3424 (2019).

16. Deng, Y. et al. Quantum anomalous Hall effect in intrinsic magnetic topological insulator $\mathrm{MnBi}_{2} \mathrm{Te}_{4}$. Science 367, 895-900 (2020).

17. Ghimire, M. P. et al. Creating Weyl nodes and controlling their energy by magnetization rotation. Phys. Rev. Res. 1, 032044 (2019).

18. Zhang, D. et al. Topological axion states in the magnetic insulator $\mathrm{MnBi}_{2} \mathrm{Te}_{4}$ with the quantized magnetoelectric effect. Phys. Rev. Lett. 122, 206401 (2019).

19. Vidal, R. C. et al. Topological electronic structure and intrinsic magnetization in $\mathrm{MnBi}_{4} \mathrm{Te}_{7}: \mathrm{a} \mathrm{Bi}_{2} \mathrm{Te}_{3}$ derivative with a periodic Mn sublattice. Phys. Rev. X 9, 041065 (2019).

20. Zou, J., He, Z. \& Xu, G. The study of magnetic topological semimetals by first principles calculations. NPJ Comput. Mater. 5, 96 (2019).

21. Liao, Z., Jiang, P., Zhong, Z. \& Li, R.-W. Materials with strong spin-textured bands. npj Quantum Mater. 5, 30 (2020).

22. Zyuzin, A. A., Wu, S. \& Burkov, A. A. Weyl semimetal with broken time reversal and inversion symmetries. Phys. Rev. B 85, 165110 (2012).

23. Chang, G. et al. Topological Hopf and chain link semimetal states and their application to $\mathrm{Co}_{2} \mathrm{MnGa}$. Phys. Rev. Lett. 119, 156401 (2017).

24. Belopolski, I. et al. Discovery of topological Weyl fermion lines and drumhead surface states in a room temperature magnet. Science 365, 1278-1281 (2019).

25. Liu, E. et al. Giant anomalous Hall effect in a ferromagnetic kagome-lattice semimetal. Nat. Phys. 14, 1125-1131 (2018).

26. Wang, Q. et al. Large intrinsic anomalous Hall effect in half-metallic ferromagnet $\mathrm{Co}_{3} \mathrm{Sn}_{2} \mathrm{~S}_{2}$ with magnetic Weyl fermions. Nat. Commun. 9, 3681 (2018).

27. Morali, N. et al. Fermi-arc diversity on surface terminations of the magnetic Weyl semimetal $\mathrm{Co}_{3} \mathrm{Sn}_{2} \mathrm{~S}_{2}$. Science 365, 1286-1291 (2019).

28. Liu, D. et al. Magnetic Weyl semimetal phase in a Kagomé crystal. Science $\mathbf{3 6 5}$, 1282-1285 (2019).

29. Soh, J.-R. et al. Ideal Weyl semimetal induced by magnetic exchange. Phys. Rev. B 100, 201102 (2019).

30. $\mathrm{Su}, \mathrm{H}$. et al. Magnetic exchange induced Weyl state in a semimetal EuCd $\mathrm{Sb}_{2}$. APL Mater. 8, 011109 (2020).

31. Tsokol', A. O., Bodak, O. I., Marusin, E. P. \& Zavdonik, V. E. X-ray diffraction studies of ternary $\mathrm{RRhC}_{2}(\mathrm{R}=\mathrm{La}, \mathrm{Ce}, \mathrm{Pr}, \mathrm{Nd}, \mathrm{Sm})$ compounds). Kristallografiya $\mathbf{3 3}, 345-348$ (1988).

32. Hoffmann, R. D., Jeitschko, W. \& Boonk, L. Structural, chemical, and physical properties of rare-earth metal rhodium carbides $\mathrm{LnRhC}_{2}(\mathrm{Ln}=\mathrm{La}, \mathrm{Ce}, \mathrm{Pr}, \mathrm{Nd}, \mathrm{Sm})$. Chem. Mater. 1, 580-586 (1989).

33. Hoffmann, R.-D., Wachtmann, K. H., Ebel, T. \& Jeitschko, W. GdRuC 2 , a ternary carbide with filled NiAs structure. J. Solid State Chem. 118, 158-162 (1995).

34. Matsuo, S. et al. Antiferromagnetism of $\mathrm{GdCoC}_{2}$ and $\mathrm{GdNiC}_{2}$ intermetallics studied by magnetization measurement and ${ }^{155} \mathrm{Gd}$ Mössbauer spectroscopy. J. Magn. Magn. Mater. 161, 255-264 (1996). 
35. Onodera, $\mathrm{H}$. et al. Magnetic properties of single-crystalline $\mathrm{RNiC}_{2}$ compounds $(\mathrm{R}$ $=\mathrm{Ce}, \mathrm{Pr}, \mathrm{Nd}$ and Sm). J. Magn. Magn. Mater. 182, 161-171 (1998).

36. Meng, L. et al. Magnetic properties and giant reversible magnetocaloric effect in $\mathrm{GdCoC}_{2}$. RSC Adv. 6, 74765-74768 (2016).

37. Meng, L., Jia, Y. \& Li, L. Large reversible magnetocaloric effect in the $\mathrm{RECoC}_{2}(\mathrm{RE}=$ Ho and Er) compounds. Intermetallics 85, 69-73 (2017).

38. Lee, W., Zeng, H., Yao, Y. \& Chen, Y. Superconductivity in the Ni based ternary carbide LaNiC 2 . Phys. C: Supercond. 266, 138-142 (1996).

39. Hirose, Y. et al. Fermi surface and superconducting properties of noncentrosymmetric LaNiC . J. Phys. Soc. Jpn. 81, 113703 (2012).

40. Hillier, A. D., Quintanilla, J. \& Cywinski, R. Evidence for time-reversal symmetry breaking in the noncentrosymmetric superconductor $\mathrm{LaNiC}_{2}$. Phys. Rev. Lett. 102, 117007 (2009).

41. Yanagisawa, T. \& Hase, I. Nonunitary triplet superconductivity in the noncentrosymmetric rare-earth compound $\mathrm{LaNiC}_{2}$. J. Phys. Soc. Jpn. 81, SB039 (2012).

42. Kolincio, K. K., Górnicka, K., Winiarski, M. J., Strychalska-Nowak, J. \& Klimczuk, T. Field-induced suppression of charge density wave in $\mathrm{GdNiC}_{2}$. Phys. Rev. B 94, 195149 (2016).

43. Steiner, $S$. et al. Single-crystal study of the charge density wave metal $\mathrm{LuNiC}_{2}$. Phys. Rev. B 97, 205115 (2018).

44. Kolincio, K. K., Roman, M. \& Klimczuk, T. Charge density wave and large nonsaturating magnetoresistance in $\mathrm{YNiC}_{2}$ and $\mathrm{LuNiC}_{2}$. Phys. Rev. B 99, 205127 (2019).

45. Hanasaki, N. et al. Successive transition in rare-earth intermetallic compound $\mathrm{GdNiC}_{2}$. J. Phys. Conf. Ser. 320, 012072 (2011).

46. Shimomura, S. et al. Charge-density-wave destruction and ferromagnetic order in $\mathrm{SmNiC}_{2}$. Phys. Rev. Lett. 102, 076404 (2009).

47. Laverock, J., Haynes, T. D., Utfeld, C. \& Dugdale, S. B. Electronic structure of $\mathrm{RNiC}_{2}$ ( $\mathrm{R}=\mathrm{Sm}, \mathrm{Gd}$, and Nd) intermetallic compounds. Phys. Rev. B 80, 125111 (2009).

48. Hanasaki, N. et al. Magnetic field switching of the charge-density-wave state in the lanthanide intermetallic $\mathrm{SmNiC}_{2}$. Phys. Rev. B 85, 092402 (2012).

49. Prathiba, G. et al. Tuning the ferromagnetic phase in the CDW compound $\mathrm{SmNiC}_{2}$ via chemical alloying. Sci. Rep. 6, 26530 (2016).

50. Kim, J. N., Lee, C. \& Shim, J.-H. Chemical and hydrostatic pressure effect on charge density waves of $\mathrm{SmNiC}_{2}$. N. J. Phys. 15, 123018 (2013).

51. Hanasaki, N. et al. Interplay between charge density wave and antiferromagnetic order in $\mathrm{GdNiC}_{2}$. Phys. Rev. B 95, 085103 (2017).

52. Perdew, J. P., Burke, K. \& Ernzerhof, M. Generalized gradient approximation made simple. Phys. Rev. Lett. 77, 3865-3868 (1996).

53. Koepernik, K. \& Eschrig, H. Full-potential nonorthogonal local-orbital minimumbasis band-structure scheme. Phys. Rev. B 59, 1743-1757 (1999).

54. Richter, M. Density functional theory applied to $4 f$ and $5 f$ elements and metallic compounds. In Buschow K. H. J. (Ed) Handbook of Magnetic Materials, Vol. 13, Chap. 2, 87-228 (Elsevier, 2001)

55. Czyzyk, M. T. \& Sawatzky, G. A. Local-density functional and on-site correlations: the electronic structure of $\mathrm{La}_{2} \mathrm{CuO}_{4}$ and $\mathrm{LaCuO}_{3}$. Phys. Rev. B 49, 14211-14228 (1994).

56. Jain, A. et al. Commentary: The materials project: a materials genome approach to accelerating materials innovation. APL Mater. 1, 011002 (2013).

57. Xu, Y. et al. Topological nodal lines and hybrid Weyl nodes in $\mathrm{YCoC}_{2}$. APL Mater. 7 , 101109 (2019).

58. Kuroda, K. et al. Evidence for magnetic Weyl fermions in a correlated metal. Nat. Mater. 16, 1090-1095 (2017).

59. Šmejkal, L., González-Hernández, R., Jungwirth, T. \& Sinova, J. Crystal time-reversal symmetry breaking and spontaneous Hall effect in collinear antiferromagnets. Sci. Adv. 6, eaaz8809 https://www.science.org/doi/abs/10.1126/sciadv.aaz8809 (2020).

60. Friedan, D. A proof of the Nielsen-Ninomiya theorem. Commun. Math. Phys. 85, 481-490 (1982)

61. Witten, E. Three lectures on topological phases of matter. Riv. Del. Nuovo Cim. 39 313-370 (2016)

62. Meng, T. \& Budich, J. C. Unpaired Weyl nodes from long-ranged interactions: fate of quantum anomalies. Phys. Rev. Lett. 122, 046402 (2019).

63. Crippa, L. et al. Nonlocal annihilation of Weyl fermions in correlated systems. Phys. Rev. Res. 2, 012023 (2020).

64. Gosálbez-Martínez, D., Souza, I. \& Vanderbilt, D. Chiral degeneracies and Fermisurface Chern numbers in bcc Fe. Phys. Rev. B 92, 085138 (2015).

65. Sato, $\mathrm{H}$. et al. Reduction of $\mathrm{Nd}$ moments and local magnetic anisotropy in $\mathrm{Nd}_{2} \mathrm{Fe}_{14} \mathrm{~B}$ single crystals. AIP Adv. 11, 025224 (2021).

66. Grössinger, R., Sun, X., Eibler, R., Buschow, K. \& Kirchmayr, H. The temperature dependence of the anisotropy field in $\mathrm{R}_{2} \mathrm{Fe}_{14} \mathrm{~B}$ compounds $\mathrm{R}=\mathrm{Y}, \mathrm{La}, \mathrm{Ce}, \mathrm{Pr}, \mathrm{Nd}$, Gd, Ho, Lu). J. Phys., Colloq. 46, C6-221-C6-224 (1985).

67. Zhong, S., Moore, J. E. \& Souza, I. Gyrotropic magnetic effect and the magnetic moment on the Fermi surface. Phys. Rev. Lett. 116, 077201 (2016).

68. Ma, J. \& Pesin, D. A. Chiral magnetic effect and natural optical activity in metals with or without Weyl points. Phys. Rev. B 92, 235205 (2015).

69. Son, D. T. \& Yamamoto, N. Berry curvature, triangle anomalies, and the chiral magnetic effect in fermi liquids. Phys. Rev. Lett. 109, 181602 (2012).
70. Kharzeev, D. E., Kikuchi, Y. \& Meyer, R. Chiral magnetic effect without chirality source in asymmetric Weyl semimetals. Eur. Phys. J. B 91, 83 (2018).

71. Kharzeev, D. E., Kikuchi, Y., Meyer, R. \& Tanizaki, Y. Giant photocurrent in asymmetric Weyl semimetals from the helical magnetic effect. Phys. Rev. B 98, 014305 (2018)

72. Kolincio, K. K., Roman, M., Winiarski, M. J., Strychalska-Nowak, J. \& Klimczuk, T. Magnetism and charge density waves in $R_{N i C}(R=C e, P r, N d)$. Phys. Rev. B 95, 235156 (2017).

73. Dorado, B. et al. Advances in first-principles modelling of point defects in $\cup_{2}: f$ electron correlations and the issue of local energy minima. J. Phys. Condens. Matter 25, 333201 (2013).

74. Allen, J. P. \& Watson, G. W. Occupation matrix control of $d$ - and f-electron localisations using DFT $+U$. Phys. Chem. Chem. Phys. 16, 21016-21031 (2014).

\section{ACKNOWLEDGEMENTS}

We thank Ulrike Nitzsche for technical assistance and Klaus Koepernik for discussion. We acknowledge financial support from the German Research Foundation (Deutsche Forschungsgemeinschaft, DFG) via SFB1143 Project No. A5 and under Germany's Excellence Strategy through Würzburg-Dresden Cluster of Excellence on Complexity and Topology in Quantum Matter-ct.qmat (EXC 2147, Project No. 390858490). R.R. and M.R. acknowledge partial financial support from the European Union (ERDF) and the Free State of Saxony via the ESF Projects No. 100231947 and No. 100339533 (Young Investigators Group Computer Simulations for Materials Design-CoSiMa) during the early stages of the project. J.I.F. acknowledges the support from the Alexander von Humboldt foundation.

\section{AUTHOR CONTRIBUTIONS}

R.R. carried out the DFT calculations. B.S. computed and analyzed the topological properties under the guidance of J.I.F. M.R. and J.v.d.B. were responsible for project planning. All the authors contributed to the analysis of results and preparation of the manuscript.

\section{FUNDING}

Open Access funding enabled and organized by Projekt DEAL.

\section{COMPETING INTERESTS}

The authors declare no competing interests.

\section{ADDITIONAL INFORMATION}

Supplementary information The online version contains supplementary material available at https://doi.org/10.1038/s41535-022-00423-z.

Correspondence and requests for materials should be addressed to Rajyavardhan Ray or Jeroen van den Brink.

Reprints and permission information is available at http://www.nature.com/ reprints

Publisher's note Springer Nature remains neutral with regard to jurisdictional claims in published maps and institutional affiliations.

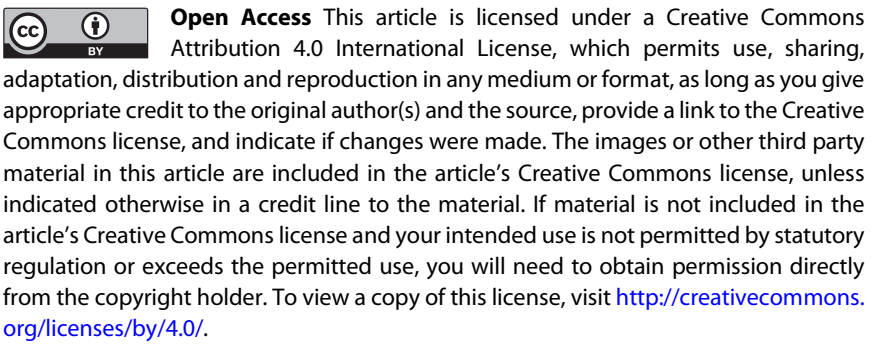

(c) The Author(s) 2022 